\title{
Topographic pattern of the brachial plexus at the axillary fossa through real-time ultrasonography in Koreans
}

\author{
Jin Hye Han, Youn Jin Kim, Jong Hak Kim, Dong Yeon Kim, Guie Yong Lee, and Chi Hyo Kim \\ Department of Anesthesiology and Pain Medicine, Ewha Womans University School of Medicine, Seoul, Korea
}

Background: The ability to explore the anatomy has improved our appreciation of the brachial anatomy and the quality of regional anesthesia. Using real-time ultrasonography, we investigated the cross-sectional anatomy of the brachial plexus and of vessels at the axillary fossa in Koreans.

Methods: One hundred and thirty-one patients scheduled to undergo surgery in the region below the elbow were enrolled after giving their informed written consent. Using the 5-12 MHz linear probe of an ultrasound system, we examined cross-sectional images of the brachial plexus in the supine position with the arm abducted by $90^{\circ}$, the shoulder externally rotated, and the forearm flexed by $90^{\circ}$ at the axillary fossa. The results of the nerve positions were expressed on a 12 -section pie chart and the numbers of arteries and veins were reported.

Results: Applying gentle pressure to prevent vein collapse, the positions of the nerves changed easily and showed a clockwise order around the axillary artery (AA). The most frequent positions were observed in the 10-11 section (79.2\%) for the median, 1-2 section (79.3\%) for the ulnar, 3-5 section (78.4\%) for the radial, and 8-9 section (86.9\%) for the musculocutaneous nerve. We also noted anatomical variations consisting of double arteries (9.2\%) and multiple axillary veins (87\%).

Conclusions: Using real-time ultrasonography, we found that the anatomical pattern of the major nerves in Koreans was about $80 \%$ of the frequent position of individual nerves, $90.8 \%$ of the single $\mathrm{AA}$, and $87 \%$ of multiple veins around the AA. (Korean J Anesthesiol 2014; 67: 310-316)

Key Words: Anatomy, Axilla, Brachial plexus, Ultrasonography.

Received: May 7, 2014. Revised: 1st, June 27, 2014; 2nd, July 2, 2014; 3rd, July 4, 2014. Accepted: July 4, 2014.

Corresponding author: Youn Jin Kim, M.D., Ph.D., Department of Anesthesiology and Pain Medicine, Ewha Womans University School of Medicine, 1071, Anyangcheon-ro, Yangcheon-gu, Seoul 158-710, Korea. Tel: 82-2-2650-5285, Fax: 82-2-2655-2924, E-mail: ankyj@ewha.ac.kr (c) This is an open-access article distributed under the terms of the Creative Commons Attribution Non-Commercial License (http:// creativecommons.org/licenses/by-nc/3.0/), which permits unrestricted non-commercial use, distribution, and reproduction in any medium, provided the original work is properly cited. 


\section{Introduction}

Axillary brachial plexus block (ABPB) is an excellent choice of anesthetic method for elbow, forearm, and hand surgery. Offering a superior pain control effect in comparison with general anesthesia, it allows for improvement of the motor function as well as quicker recovery and discharge [1]. In addition, when compared with other types of brachial plexus block, ABPB can be performed relatively easily, making it the most frequently used method as it avoids the risk of pneumothorax or diaphragm paralysis by phrenic nerve palsy [2].

Various methods have been used to increase the success rate of $\mathrm{ABPB}$ and to decrease $\mathrm{ABPB}$ complications. In the past, the fascial click technique, a blind technique, as well as the paresthesia and transarterial techniques were used. Later, nerve stimulator-guided technique was introduced to verify the degree of proximity between the needle and the target nerve. However, the individual anatomical variations and compartments of nerves and blood vessels hinder drug diffusion, meaning that the brachial plexus block is performed incompletely when using this method [3]. In fact, the success rates of the nerve stimulator-guided techniques vary from 54 to $90 \%$ [4-6]. Recently, the use of ultrasound (US)-guided nerve block for peripheral nerve block has been developed and steadily extended. US-guided nerve block makes it possible to distinguish the positions of the block needle, of the nerves, and of the important surrounding structures through ultrasonic images in order to identify them and to verify the real-time diffusion pattern of the local anesthetic (LA). As a result, an effective nerve block may be induced with a small dose of LA. Nevertheless, basic anatomical knowledge as well as variations in the ultrasonic image interpretation capabilities of individual anesthesiologists may have a significant effect on the success rate of this technique [7].

Retzl et al. [8] reported the anatomical positions of the three main nerves of the axillary brachial plexus, which are the median nerve, the ulnar nerve, and the radial nerve, by using ultrasonography. Christophe et al. [9] reported the anatomical positions and variations of the musculocutaneous (MC) nerve as well as of the three main nerves. Such reports on anatomical positions and variations have enhanced our anatomical understanding and enabled effective regional anesthesia during ABPB. However, there have been few anatomical reports on the axillary brachial plexus of Koreans. Besides, the previous studies conducted through ultrasonography focused on the anatomical positions of the axillary brachial plexus relative to the axillary artery (AA) in the axillary fossa. It is presumed that anatomical understanding and verification of not only the four main nerves in the axillary brachial plexus, but also of the veins and arteries, which are the main surrounding structures during the USguided nerve block, may offer effective brachial plexus blockade help by reducing the potential complications caused by needle manipulation.

With this in mind, using US guidance, this study investigated the relative anatomical pattern of the four main nerves of the axillary brachial plexus in the axillary fossa around the AA, as well as the distribution of blood vessels in Korean patients undergoing arm surgery under regional anesthesia.

\section{Materials and Methods}

Among the patients scheduled to undergo surgery for the hand, wrist, or forearm under regional anesthesia, 131 were selected as the subjects of this study. Following the approval of the Institutional Review Board, these patients were given an explanation of the purposes and methods of this study, and subsequently submitted their written consent. The subjects were American Society of Anesthesiologists Physical Status Class I-II adult patients aged 20-85. Pregnant women and patients who had an infection in an injection region, who were allergic to a LA, or who had a hemostatic disorder, as well as those who were unable to communicate with a doctor or who had difficulty in making a positional change of the arm to be blocked due to pain or limited motion of the elbow or of the arm articulation, were excluded from the study.

The nerve block was performed by two expert anesthesiologists who had performed US guided-ABPB 50 times or over. Upon the patient's arrival in the operating room, standard monitoring including electrocardiography, non-invasive blood pressure, and pulse oximetry was initiated. Patients lay in the supine position, and their arm was externally rotated with a $90^{\circ}$ shoulder abduction and a $90^{\circ}$ elbow flexion. Oxygen $(6 \mathrm{~L} / \mathrm{min})$ was supplied through a mask, and the patients were sedated with intravenous injections of midazolam (1-2 mg) and fentanyl (25$50 \mu \mathrm{g})$. The axillary area was disinfected with a povidone-iodine solution. The positions of the median nerve, the ulnar nerve, the radial nerve, and the MC nerve - which are the branches of the peripheral nerves of the axillary brachial plexus - as well as those of the blood vessels, were identified using the Sonosite M-Turbo ultrasonography instrument (Sonosite ${ }^{\circledR}$, Bothell, WA, USA). A $38 \mathrm{~mm}$ and 7-12 $\mathrm{MHz}$ linear ultrasonic transducer probe was wrapped in sterilized transparent tape and applied to the axillary fossa bounded by the anterior and posterior axillary fold, with the probe upwards so that the blood vessels and nerves could be viewed on the short axis plane. The probe was positioned so that the AA could be viewed at the center or in the $1 / 3$ right area of the US image, and the 3 oclock direction may be placed in the medial direction and the 9 oclock direction in the lateral direction with respect to the AA. We distinguished the arteries from the veins by their darker and more defined circular structure and by their lack of easy compressibility, as 
well as with a color Doppler and pulsation using real-time US [10]. At that point, the side of the probe closest to the skin, which is in the 12 oclock direction, faces the anterior direction, while the deepest side of the probe faces the posterior direc-

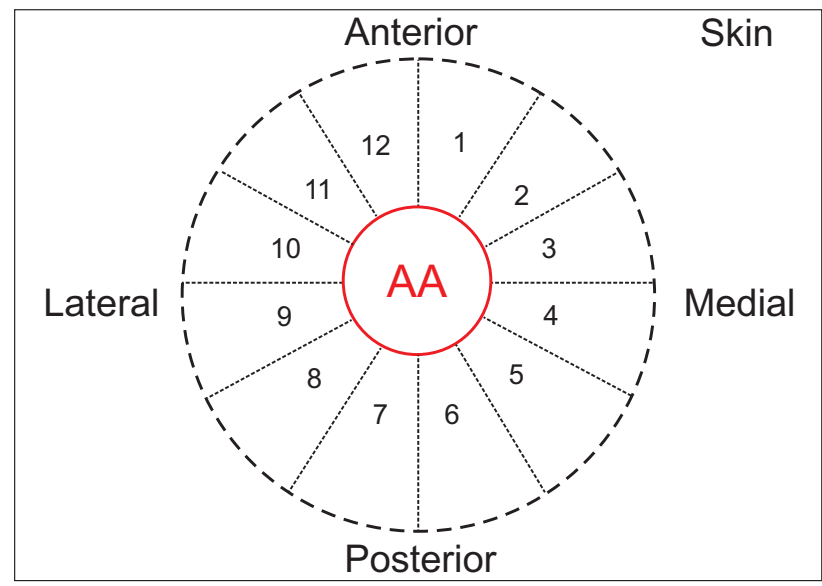

Fig. 1. Schematic illustration of cross-section ultrasound scan image with ultrasound probe placed at the axillary fossa. AA: axillary artery. tion. The probe was fixed at the axillary fossa to identify the AA and veins as well as the nerves, which have a honeycomb shape mixing circles and ovals around the AA [10]. The numbers of the AA and veins were recorded. Afterwards, a gentle pressure was exerted on the probe to the extent that the axillary veins could not be viewed, and the positions of the median nerve, the ulnar nerve, the radial nerve, and the MC nerve, which are the branches of the peripheral nerves of the axillary brachial plexus, were recorded. The position of each of the nerves was recorded using the pie chart with 12 divisions previously employed by Christophe et al. [9] (Figs. 1 and 2). When a nerve extended to two or more divisions, the nerve position was recorded as the division in which the greater portion of the nerve was included.

Subsequently, a 22-gauge, $50 \mathrm{~mm}$ needle (Stimuplex ${ }^{\circledR}, \mathrm{B}$. Braun, Melsungen, Germany) was used to block the nerve using the in-plane technique. After placing the nerve block needle in a position close to the target nerve identified in the US image, a nerve stimulator (Stimuplex ${ }^{\circledR}$ Dig RC, B. Braun, Melsungen, Germany) was used to give a stimulation of $0.5-0.6 \mathrm{~mA}$ and $2 \mathrm{~Hz}$ to verify whether the nerve showed a motor reaction. A LA of 5 to $8 \mathrm{ml}$ of $1.5 \%$ lidocaine was injected into each of the
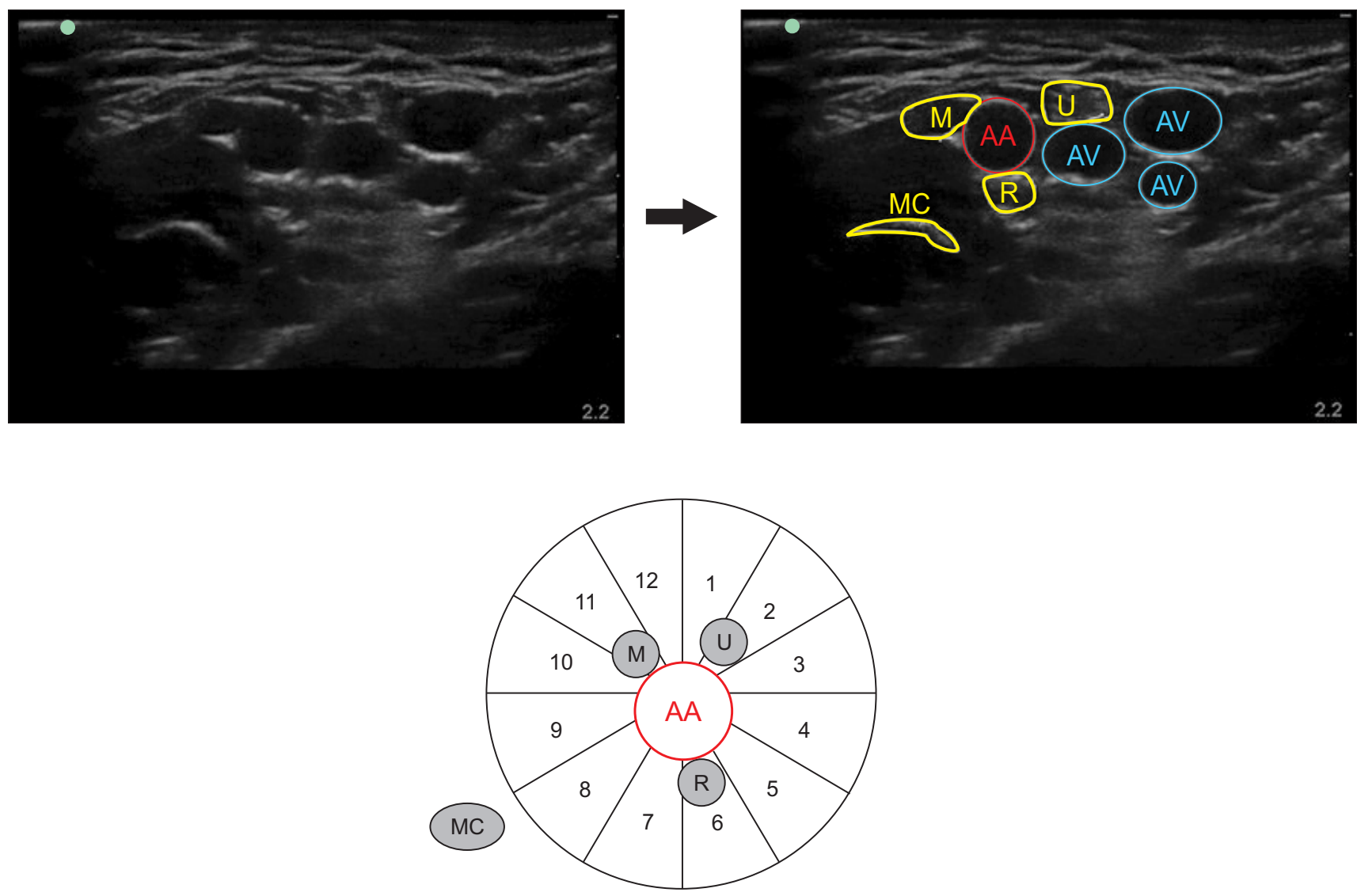

Fig. 2. Positions of the individual nerves observed in clockwise order around the AA using ultrasonography in 131 Koreans. AA: axillary artery, AV: axillary vein, $\mathrm{M}$ : median nerve, $\mathrm{U}$ : ulnar nerve, R: radial nerve, MC: musculocutaneous nerve. 
nerves. When the lidocaine was injected, the hypoechoic LA surroundings around the nerve (doughnut sign) were observed on a real-time US image. If they could not be observed, the needle was repositioned.

The data were analyzed using SPSS version 20.0 (SPSS Inc, Chicago, IL, USA) and were expressed as a mean with the SD or frequency and percentages for categorical variables.

\section{Results}

The demographic data of the 131 patients are shown in Table 1. In all patients, when the AA was located at the center or in the $1 / 3$ right area of the US image, the median, ulnar, radial, and MC nerves were observed in clockwise order within 1.8 to 2.7

Table 1. Demographic Data

\begin{tabular}{lc}
\hline & $\mathrm{N}=131$ \\
\hline Age $(\mathrm{yr})$ & $46.8 \pm 12.2$ \\
Weight $(\mathrm{kg})$ & $64.2 \pm 13.1$ \\
Height $(\mathrm{cm})$ & $164.6 \pm 7.0$ \\
BMI $\left(\mathrm{kg} / \mathrm{m}^{2}\right)$ & $23.2 \pm 2.8$ \\
Sex $(\mathrm{M} / \mathrm{F})$ & $78 / 55$ \\
ASA classification $(1 / 2)$ & $64 / 69$ \\
HTN & 28 \\
DM & 6 \\
\hline
\end{tabular}

Data are expressed as mean \pm SD or count. BMI: body mass index, ASA: American Society of Anesthesiologists, HTN: hypertension, DM: diabetes mellitus. $\mathrm{cm}$ of the skin. The divisions in which the nerves were most frequently found were as follows: the median nerve was found $79.2 \%$ of the time in divisions 10 to 11 , the ulnar nerve was found $79.3 \%$ of the time in divisions 1 to 2 , the radial nerve was found $78.4 \%$ of the time in divisions 3 to 5 , and the MC nerve was found $86.9 \%$ of the time in divisions 8 to 9 . However, the MC nerves were observed between the biceps muscle and the coracobrachialis muscle in divisions 8 to 9 apart from the AA (Fig. 3).

In 12 patients (9.2\% of the total subjects), two AAs were observed on the US image. Although the accessory AA was smaller than the main $\mathrm{AA}$, it was found to be close to the main AA and to extend in parallel with the main AA into the deeper region. In 109 patients ( $87 \%$ of the total subjects), two or more axillary veins were observed in a region close to a nerve around the AA (Table 2, Fig. 4).

\section{Discussion}

Understanding the anatomical structure of the axilla is a key factor to the success of ABPB. The anatomical understanding of the axilla region based on cadaveric anatomy may be different in terms of nerve positions to that of actual patients, due to the lowered tissue elasticity caused by drug treatments, changes in the volume of the blood vessels close to a nerve, and the removal of fat $[9,11]$. Similarly, the anatomical understanding of the axillary brachial plexus through MRI or CT may not reflect the actual nerve positions, given that the arm position is different
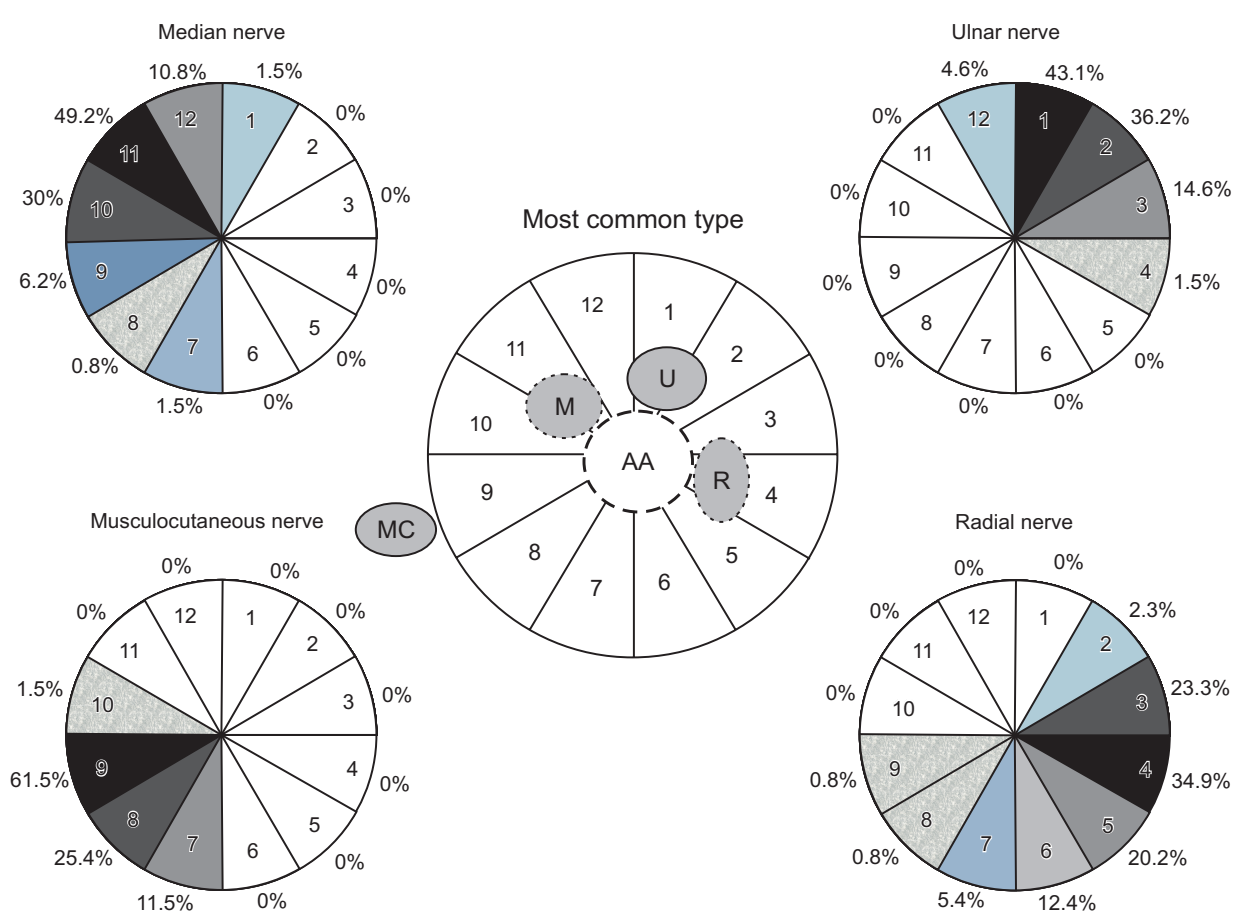

Fig. 3. Schematic representation of common arrangements of the four individual nerves of the brachial plexus as confirmed by ultrasonography in 131 Koreans. AA: axillary artery, AV: axillary vein, M: median nerve, U: ulnar nerve, R: radial nerve, $\mathrm{MC}$ : musculocutaneous nerve. 
from that assumed in a clinical nerve block [11-13]. It has been reported that when the arm is externally rotated by $90^{\circ}$ at the shoulder region, in a normal brachial plexus the median nerve is generally located on the lateral side of the AA, the ulnar nerve is located in the anteromedial position, and the radial nerve is located in the posterior position $[2,14]$. Recent anatomical studies using ultrasonography not only showed results that were similar

Table 2. Numbers of Axillary Arteries and Veins Observed in the Axillary Fossa using Real-Time Ultrasound

\begin{tabular}{cc}
\hline & $\mathrm{N}=131$ \\
\hline Axillary artery & $119(90.8 \%)$ \\
1 & $12(9.2 \%)$ \\
2 & \\
Axillary Vein & $22(13.0 \%)$ \\
1 & $56(42.7 \%)$ \\
2 & $33(25.1 \%)$ \\
3 & $15(11.5 \%)$ \\
4 & $5(3.8 \%)$ \\
5
\end{tabular}

Data are expressed as count (percentage). to those of previous studies, but also revealed the existence of diverse anatomical variations $[8,9,14-16]$. In a study conducted with an $8 \mathrm{MHz}$ US probe, Retz et al. [8] reported that the ulnar nerve was found in the anteromedial position in as little as $59 \%$ of cases, that there existed diverse anatomical variations of the ulnar nerve, and that the radial nerve was positioned not in the posterior region but in the posteromedial region in more than half of the cases. It was also reported that the positions of the nerves are not fixed but are easily altered when a slight pressure is imposed on the skin by the US probe. In addition, it was reported that when moving from the axillary region to the distal region, individual peripheral nerve branches are branched out from the original branch and grow farther from the AA region, producing more anatomical variations; thus, nerve blocks should be performed close to the axilla in order to decrease the risk of anatomical variations and to increase the success rate.

We identified the relationship between the positions of the AA and the nerves, finding that the topographic anatomy was similar to that evidenced in Christophe et al. [9]. The median nerve, ulnar nerve, radial nerve, and MC nerve were observed
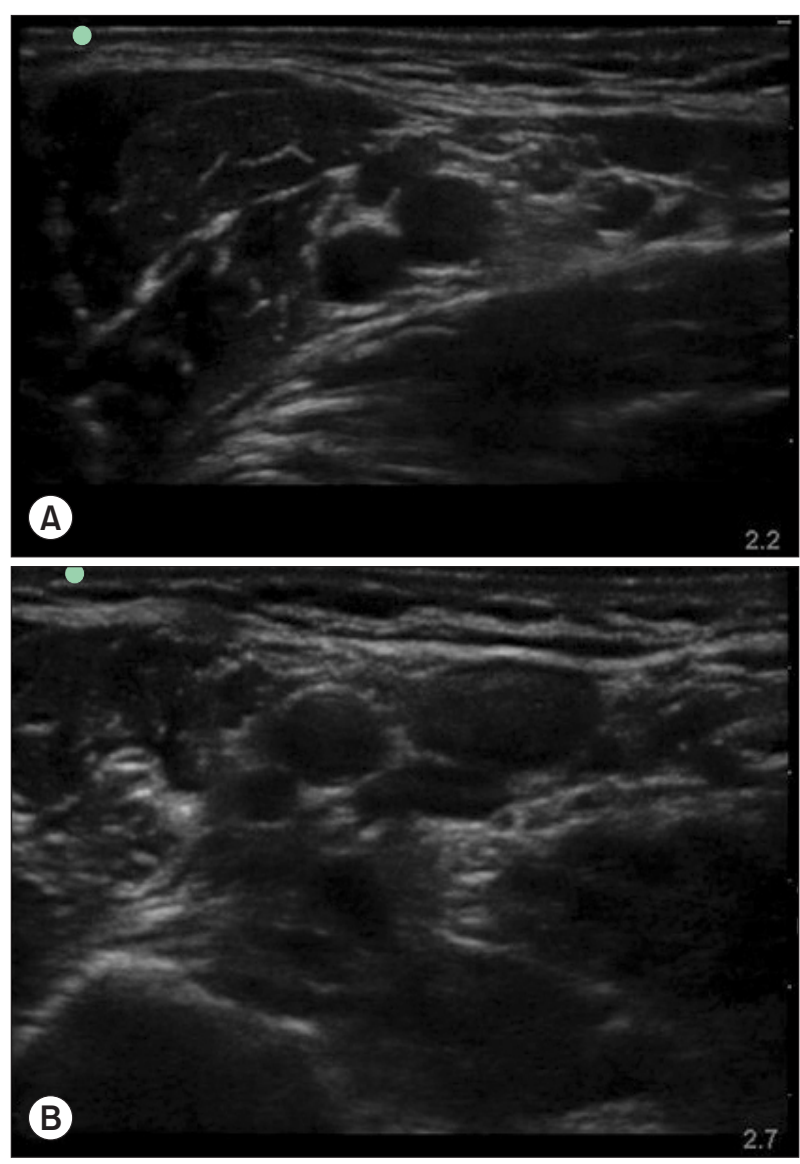
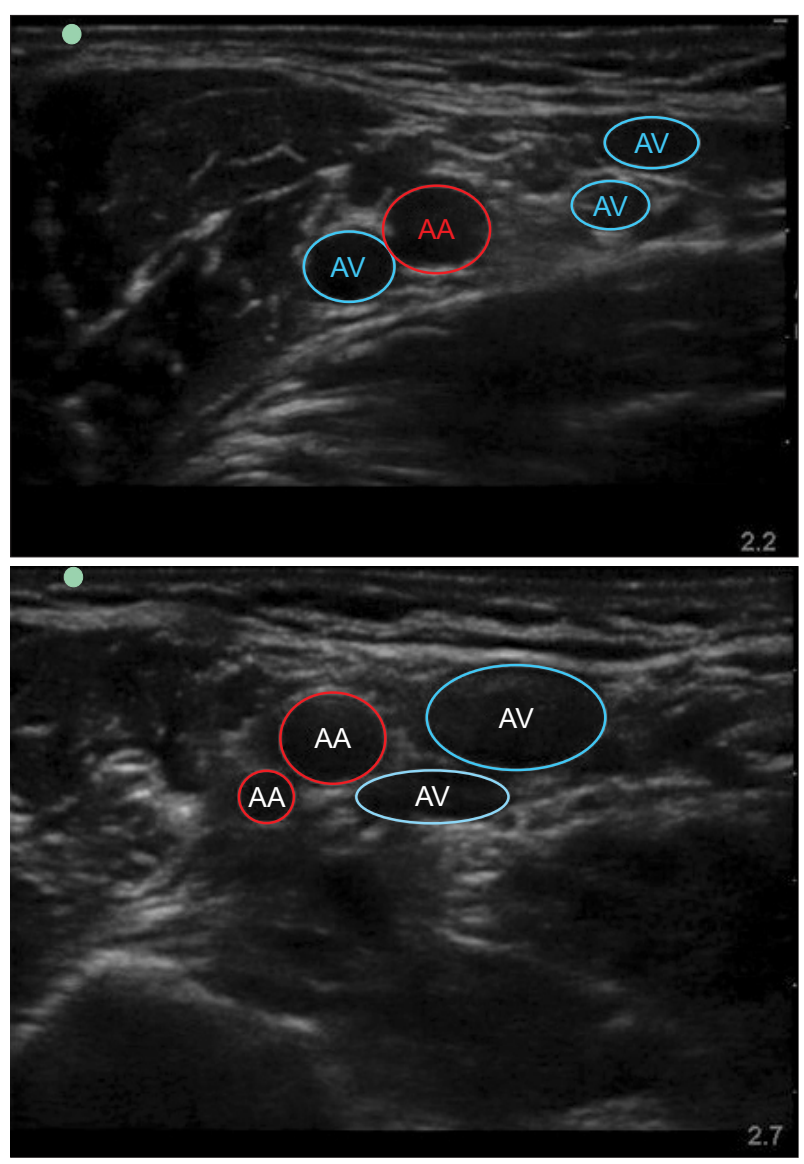

Fig. 4. Axillary vascular variations as confirmed by cross-sectional ultrasound scan image at the axillary fossa. (A) multiple axillary veins, (B) double axillary arteries. AA: axillary artery, AV: axillary vein. 
in clockwise order around the AA, and the divisions in which the nerves were most frequently found were as follows: the median nerve was found $79.2 \%$ of the time in divisions 10 to 11 , the ulnar nerve was found $79.3 \%$ of the time in divisions 1 to 2 , the radial nerve was found $78.4 \%$ of the time in divisions 3 to 5 , and the MC nerve was found $86.9 \%$ of the time in divisions 8 to 9 .

The MC nerve was most often found outside the neurovascular bundle. Among the anatomical variations of the axillary brachial plexus, variations of the MC nerve have been reported most frequently. The most frequent anatomical variations reported by Christophe et al. [9], namely, the MC nerve being positioned near the AA (in 13\% of the patients), and the inclusion of the median nerves in the MC nerve bundles (in 5.2\% of the patients), were not found in this study. Prasada Rao and Chaudhary [17] reported that no MC nerves were found in $8 \%$ of cadaveric anatomy cases. Venieratos and Anagnostopoulou [18] reported a connection of the MC nerve with the median nerve in $20 \%$ of their patients. Choi et al. [15] also reported an anatomical variation characterized by a connection of the MC nerve to the median nerve in 3 to $5 \%$ of their patients, asserting that the cause of the connection may be the fusion of both nerves or the presence of one supplementary branch between both nerves. In cases in which the MC nerve is not easily identified on an US image, anatomical variations in which the MC nerve is connected or fused with the median nerve may be taken into consideration. However, in this study, no case arose in which the MC nerve was not observed. In all cases, the MC nerve was observed as oval or flat on the hyperechoic plane between the biceps muscle and the coracobrachialis muscle apart from the AA. Anatomical variations of the MC nerve were not observed in our study. This may have been because the number of subjects was small, because the ultrasound probe was placed in a more proximal region, or perhaps due to racial differences. Further study involving a larger number of clinical observations is needed in relation to this finding.

Abnormal vascular formations in the axillary region are common and have been reported as an important factor to the success of ABPB [19-23]. Kutiyanawal et al. [20] reported that $21 \%$ of their subjects presented abnormal venous structures, including two axillary veins in $10 \%$ of the subjects. Uglietta and Kadir [21] evaluated anomalies through arteriograms of the upper extremities of 100 patients. They also reported that $9 \%$ of the subjects presented a variant type of arterial anatomy, such as a higher origin of the radial artery. Anatomical variations of the AA, two axillary arteries, a bifida brachial artery, and a superficial and deep brachial artery were observed in $10 \%$ of the subjects [23]. Christophe et al. [9] reported that two axillary arteries were observed in $4.5 \%$ of their subjects. In their study, deep axillary arteries affected the anatomical positions of the nerves. It was observed that all branches of the ulnar, radial, and median nerves appeared as a single mass in the posteromedial region between the two axillary arteries. In our study, two axillary arteries were identified in $9.2 \%$ of the subjects, echoing the findings of previous studies. However, in contrast with the results of Christophe et al. [9], we did not find a case in which a single mass appeared to affect the running and position of each of the peripheral nerve branches due to the results of an abnormal variation of two axillary arteries.

We observed two or more abnormal positions of the veins in $87 \%$ of the subjects. Studies in which abnormal veins are observed are rare, and there are few reports of the effect of the number of abnormal veins on ABPB [14,20]. In our study, two or more veins, and even three to five veins in some cases, were observed in a position adjacent to an AA. Veins were observed between an AA and nerves in a number of cases, while in others they were found in a region close to each nerve apart from the AA. These types of multiple veins and different positions may affect the nerve block, as veins of different sizes move the nerves to various positions. In the case of the anatomical variation found in the study by Christophe et al. [9], where two or three neighboring nerves had been observed as a mass but were later separated from each other by injecting a local anesthetic, multiple axillary veins existing in a region close to the AA caused the nerves to be positioned in a region away from the artery. The veins may be easily collapsed through pressure exerted on the skin by the US probe, which is used to locate each of the nerves in a position close to the AA, such that the individual nerves may be incorrectly perceived to be positioned closely to one another. Therefore, it is presumed that the anatomical variation may be occurring not in one of the axillary nerves, but instead in the abnormal axillary veins. When a nerve is positioned away from the AA, more time may be required for a local anesthetic to be diffused than for a nerve located close to the AA. Multiple veins may also interfere with the procedure, especially when they are located between the AA and a nerve. As a result, the onset of the local anesthetic effect may be delayed and a clinically incomplete nerve block may occur. Moreover, unless the blood is aspirated frequently during the anesthetic injection with strong pressure to the extent that the numbers or positions of the veins cannot be identified, it is highly probable that an unintentional intravascular injection of a local anesthetic may cause toxicity or a complication such as a hematoma. In addition, in USguided $\mathrm{ABPB}$ using multiple injections or perineural injection techniques, it is difficult to adjust the needle position close to a nerve due to abnormal blood vessels, leading to possible tissue damage and/or patient discomfort. Therefore, during US-guided ABPB performed on a patient with abnormal axillary veins, the drug diffusion pattern should be verified in real time in order to increase the block success rate and to decrease complications.

In conclusion, the anatomical positions of the peripheral 
nerve branches of the brachial plexus and of the surrounding blood vessels in Korean subjects were observed at the axillary fossa through ultrasonography. The results were similar to those of other studies, except for the finding of variations of the MC nerve and in the number of axillary veins. This anatomical analysis may help verify the position of each of the nerves and those of abnormal blood vessels during US-guided ABPB.

\section{References}

1. Chan VW, Peng PW, Kaszas Z, Middleton WJ, Muni R, Anastakis DG, et al. A comparative study of general anesthesia, intravenous regional anesthesia, and axillary block for outpatient hand surgery: clinical outcome and cost analysis. Anesth Analg 2001; 93: 1181-4.

2. Hadzic A, Carrera A, Clark TB, Gadsden J, Karamaker MK, Sala-Blanch X, et al. Hadzic's peripheral nerve blocks and anatomy for ultrasound-guided regional anesthesia. 2nd ed. New York. McGraw-Hill. 2004, pp 377-84.

3. Thompson GE, Rorie DK. Functional anatomy of the brachial plexus sheaths. Anesthesiology 1983; 59: 117-22.

4. De Tran QH, Clemente A, Doan J, Finlayson RJ. Brachial plexus blocks: a review of approaches and techniques. Can J Anaesth 2007; 54: 662-74.

5. Koscielniak-Nielsen ZJ, Nielsen PR, Nielsen SL, Gardi T, Hermann C. Comparison of transarterial and multiple nerve stimulation techniques for axillary block using a high dose of mepivacaine with adrenaline. Acta Anaesthesiol Scand 1999; 43: 398-404.

6. Sia S, Bartoli M, Lepri A, Marchini O, Ponsecchi P. Multiple-injection axillary brachial plexus block: A comparison of two methods of nerve localization-nerve stimulation versus paresthesia. Anesth Analg 2000; 91: 647-51.

7. De Andrés J, Sala-Blanch X. Ultrasound in the practice of brachial plexus anesthesia. Reg Anesth Pain Med 2002; 27: 77-89.

8. Retzl G, Kapral S, Greher M, Mauritz W. Ultrasonographic findings of the axillary part of the brachial plexus. Anesth Analg 2001; 92: 1271-5.

9. Christophe JL, Berthier F, Boillot A, Tatu L, Viennet A, Boichut N, et al. Assessment of topographic brachial plexus nerves variations at the axilla using ultrasonography. Br J Anaesth 2009; 103: 606-12.

10. Tsui B, Chan V, Finucane B, Grau T, Walji A. Atlas of ultrasound and nerve stimulation-guided regional anesthesia. New York. Springer. 2007, pp 26-9.

11. Partridge BL, Katz J, Benirschke K. Functional anatomy of the brachial plexus sheath: implications for anesthesia. Anesthesiology 1987; 66: 743-7.

12. Gerevini S, Mandelli C, Cadioli M, Scotti G. Diagnostic value and surgical implications of the magnetic resonance imaging in the management of adult patients with brachial plexus pathologies. Surg Radiol Anat 2008; 30: 91-101.

13. Hergan K, Morrigl B, Kathrein A, Buchberger W, Judmaier W, Peer S, et al. MR and CT anatomy of the axilla. Acta Radiol 1997; 38: 198-205.

14. Berthier F, Lepage D, Henry Y, Vuillier F, Christophe JL, Boillot A, et al. Anatomical basis for US-guided regional anaesthesia at the junction of the axilla and the upper arm. Surg Radiol Anat 2010; 32: 299-304.

15. Choi D, Rodríguez-Niedenführ M, Vázquez T, Parkin I, Sañudo JR. Patterns of connections between the MC and median nerves in the axilla and arm. Clin Anat 2002; 15: 11-7.

16. Chan VW, Perlas A, McCartney CJ, Brull R, Xu D, Abbas S. US guidance improves success rate of axillary brachial plexus block. Can J Anaesth 2007; 54: 176-82.

17. Prasada Rao PV, Chaudhary SC. Absence of MC nerve: two case reports. Clin Anat 2001; 14: 31-5.

18. Venieratos D, Anagnostopoulou S. Classification of communications between the musculocutaneous and median nerves. Clin Anat 1998; 11: 327-31.

19. Orebaugh SL, Williams BA. Brachial plexus anatomy: normal and variant. ScientificWorldJournal 2009; 9: 300-12.

20. Kutiyanawala MA, Stotter A, Windle R. Anatomical variants during axillary dissection. Br J Surg 1998; 85: 393-4.

21. Uglietta JP, Kadir S. Arteriographic study of variant arterial anatomy of the upper extremities. Cardiovasc Intervent Radiol 1989; 12: 145-8.

22. Bigeleisen PE. The bifid axillary artery. J Clin Anesth 2004; 16: 224-5.

23. Cavdar S, Zeybek A, Bayramiçli M. Rare variation of the axillary artery. Clin Anat 2000; 13: 66-8. 